\title{
Role of a Nurse in COVID-19 Pandemic
}

\author{
Ranjana Premnath Sharmaํ, Savitha Basiram Pohekar², Ruchira Shirkant Ankar ${ }^{3}$
}

\begin{abstract}
${ }^{1}$ Department of Medical Surgical Nursing, Smt. Radhikabai Meghe Memorial College of Nursing, Datta Meghe Institute of Medical Sciences (Deemed to Be University) Sawangi (Meghe) Wardha, Maharashtra, India. ${ }^{2}$ Department of Medical Surgical Nursing, Smt. Radhikabai Meghe Memorial College of Nursing, Datta Meghe Institute of Medical Sciences (Deemed to Be University) Sawangi (Meghe) Wardha, Maharashtra, India.. ${ }^{3}$ Department of Medical Surgical Nursing, Smt. Radhikabai

Meghe Memorial College of Nursing, Datta Meghe Institute of Medical Sciences (Deemed to Be University) Sawangi (Meghe) Wardha, Maharashtra, India.
\end{abstract}

\section{ABSTRACT}

\section{BACKGROUND}

The role of nurse changes as the health care is needed in the hospitals, society or community. Nurses are providing essential health care services throughout the health care system. In response to COVID-19 pandemic, the role of nurse changes to care or respond to the needs of the patients, their families and their caregivers. They also should take part in policies making, doing procedures and taking care of necessary supply of the material and equipment in the hospitals. All over the world nurses are demonstrating their kindness, care, courage, values and professional responsibility as nursing personnel. Prior to the COVID-19 pandemic in many institutions / organizations, there was a focus on nurses, mental health, physical health and wellbeing such as workload, anxiety, anger, irritability, burnout etc. They have to follow the rules, regulations, ethics and standard of nursing. However, while working in the isolation units or intensive care unit with COVID-19 patients, the nurse has to decide how much quality care they can provide to the patients while taking care of themselves. Hospital authorities have the responsibility to provide optimal work environments for all staff involved in the care of COVID-19 patients and health care professionals must follow the protocols that protect their ethical rights as a health care professional. In a 2018 policy brief, the American Nurses Association, said "nurse leaders are the key to preventing and containing widespread illnesses. They have the skills and education to develop coordinated global networking and properly identifying of infectious diseases".

\section{KEY WORDS}

Role of the Nurse, Covid-19, Pandemic, Professional Responsibility
Corresponding Author: Ms. Ranjana Premnath Sharma, Department of Medical Surgical Nursing, Smt. Radhikabai Meghe Memorial College of Nursing, Sawangi (Meghe) Wardha, Maharashtra, India.

E-mail:

ranjanasharma1234@rediffmail.com

DOI: $10.14260 /$ jemds/2020/554

How to Cite This Article:

Sharma RP, Pohekar SB, Ankar RS. Role of a nurse in COVID-19 pandemic. J Evolution Med Dent Sci 2020;9(35):2550-2555, DOI: $10.14260 /$ jemds/2020/554

Submission 29-05-2020,

Peer Review 22-07-2020,

Acceptance 29-07-2020,

Published 31-08-2020.

Copyright (C) 2020 JEMDS. This is an open access article distributed under Creative Commons Attribution License [Attribution 4.0 International (CC BY 4.0)] 


\section{BACKGROUND}

"The nurses' role in a pandemic begins even before a disease has an opportunity to cause widespread devastation, the World Health Organization (WHO), the American Nurses Association (ANA) and other healthcare organizations said"1. During the COVID-19 pandemic, a full lockdown is forced, means closure of schools, colleges, universities, market and workplace to prevent and protect the health of the population. It was also instructed to all stay at home only, when required to leave the home to buy the basic things. However the health care professionals have to perform their duty to serve the vulnerable and needy peoples. Among those health care professionals the nurses are the largest leading personnel within the society, to provide care during health related crisis.

Nurses' are playing very important roles, when dealing with the COVID-19 patients because they are the one who is performing the screenings, implementing triage, proving care to the patients, communicating with the caregivers, proving health education on prevention and protection from coronavirus and handling the critical conditions.

In 2019 annual report, the WHO said the world is not adequately prepared for a global health crisis. Since pandemic outbreaks are unpredictable, global health agencies have to develop plans that will provide appropriate and timely responses, the WHO said. Among those who must be involved in planning are the nurses, the organization said. Indeed, nurses around the world are filling roles to assist in the corona virus response ${ }^{1}$.

In the United Kingdom, the government is considering recalling recently retired nurses and other providers. Closer to home, the Association of camp nursing posted official guidelines for spring break camps, including having camp managers and nurses monitor children and staff for illnesses. ${ }^{1}$

At the same time, the Washington State Nurses Association is calling for local and state officials to better protect staff nurses who are working directly with sick patients. ${ }^{1}$

\section{Role of Nurses in the Screening, Triage, and Standard Precaution}

Nurse should familiar with scientific updates of COVID-19 disease and use of appropriate methods to assess, categorize the triage system, different test and management of patients. ${ }^{2}$ While screening the individual the nurses should take the following information from the person's risk for COVID-19: sign and symptoms of respiratory tract infections, travel history of the last few days and exposure to someone who has been confirmed to have COVID-19. After screening process the nurses should take the decision the person should be tested for COVID-19 or not.

Every organization is having there a standard operating procedure for triage to COVID-19 patients. The nurses should to know and review the triage policies and procedures of their organization and follow the same in response to COVID-19. The nurse role in triage is not to diagnose, but to recognize or identify the patients for separation or isolation if suspected to have COVID-19. Educate the patients to maintain respiratory hygiene and cough etiquette if that has not been done by the patient's, such as provide alcohol-based hand sanitizer, tissue paper and face mask.
Promotion of safety and prevention of transmission of infection in health care, the one should know the minimum level of precautions that is standard precautions while providing care of all patients admitted in hospital or community based. Learn and implement all health care activities to protect themselves, determine infection control measures to prevent respiratory infection such as respiratory hygiene and cough etiquette. Standard precautions like proper hand washing and the use of personal protective equipment (PPE) is necessary to apply in all areas of health care services. It also includes prevention from the needle stick injury, injury from the sharp instruments, proper waste management, cleaning, sterilization and disinfection of equipment and cleaning of the environment. ${ }^{3}$

\section{Role of Nurses in Protecting Herself from Risks}

Minimize face to face contact with patients whenever possible. Protect themselves against risk one of the best things is to use personal protective equipment while taking care of positive cases and systematic handwashing. It also includes changing out of work attire before entering into the home and showering as soon as getting inside the home. If required isolating themselves from the family members, to protect the family members as best they can. ${ }^{4}$ Proper hygiene is one of the very important factors in turning the tide of a pandemic. It is very necessary for the nurses to abide with the hygiene rules and procedures, because they work so closely and regularly with patients. ${ }^{5}$

\section{Role of Nurses in Physical Care}

Nurses' role is critical to the entire health care operation and pandemic response. They have to perform the task in the assessment of patient, triage and quarantine the patients. They can perform this task efficiently because they are having the experience of working directly with patients. Nurses are identifying the patients whose test positive or show symptoms of COVID-19 are quickly moving to quarantine or isolating, protecting other patients who may not have the disease ${ }^{5}$. The nurse should provide nursing care according to the severity of diseases. They have to understand the patients with mild, moderate and severe infection of symptoms accordingly, they have to play their role in the management of symptoms, monitoring of the disease progression, monitoring of nutrition and education related to exercise, psychological support and lifestyle changes. If the patients are critically ill they have to assist to the physician in artificial airway management and life support management. However, patients with urinary catheterization they have to provide catheter care to prevent from urinary tract infection, to bed ridden patients they have to provide back care as needed.

\section{Role of Nurses in Giving Psychological Support}

Provide psychological support to the patients and family, to help them to alleviate fear, anxiety and depression about the COVID-19. Explain to the patient, family and caregivers about the causes, risk, management and prognosis of COVID-19, so they can participate in the treatment plan. COVID-19 can cause sudden breathlessness for patients, therefore, advise them about the breathing techniques, proper ventilation, and 
warmness of room and positioning ${ }^{6}$. To find opportunities to deliver hopeful stories and positive images of local people who have suffered from the COVID-197. The risk of corona virus infection (COVID-19) may cause psychosocial problem for nurses and medical staffs. To cope with this psychosocial problem of nurses, the in-charges or head nurse should assign the areas properly who will in the isolation area and aware them about the equipment, supplies and resources of the hospital, the supervisor who will be helping will provide instant help whenever necessary. ${ }^{8}$

\section{Leadership Role of Nurses}

The head nurse or superior should provide the positive working environment for nurses to report all incidents related to COVID-19 positive patients, such as exposures to respiratory system secretion, blood products and body fluid, such incidences occur to adopt the measures for immediate follow up and support to victims ${ }^{2}$. It is the responsibility of the leader or head nurse to protect all the staffs from psychosocial problems (stress and poor mental health) during this response, so the staffs are able to perform to fulfil their roles efficiently, while performing the patient care. It is also a responsibility of head nurse to provide updated information to all staffs and rotation of staffs from higher-stress to lowerstress areas. Initiate, encourage and monitor work breaks. Make sure all staffs are aware of the facilities of mental health and psychological support services. ${ }^{7}$

\section{Role of Nurses in the Management of Equipment and Supplies}

Inadequate supply of personal protective equipment (gloves, mask, gown and eye shield) and medical supplies in the health care system can cause the spread of infection. The spread of COVID - 19 is mainly through respiratory droplets. At the time of a pandemic, it is very important to maintain the supplies of medical equipment and protective items such as masks, gloves, and hand sanitizer. These supplies can easily become short during crisis; nurses play a vital role in safeguarding these supplies from unnecessary use, theft or hoarding 5 . They are responsible for keeping an adequate amount of equipment and supplies in the isolation unit and intensive care units. Periodically they have to check that equipment and supplies are in good conditions before using for patients. Put in a requisition for necessary equipment for repair and maintenance when needed. Make sure that equipment and supplies are where it is needed to be keep there only it is kept. Make sure that all the personnel on the ward should clearly know who may use ward articles and equipment and who assumes responsibility for it. The head nurse must be vigilant and prevent waste or misuse by educating the staff in the economical and appropriate use of all equipment and materials. She may sometimes arrange in the ward class to enable the staff to know the cost of the equipment and materials.

\section{Role of Nurses in Taking Care of Pregnant Women}

The nurse should understand and trained to provide obstetric care and enough competent to implementing in recommended infection control interventions. Pregnant women with known or suspected case of COVID-19 should inform to the obstetric unit/department prior to arrival of the pregnant patients. So the necessary arrangements such as appropriate infection control preparations, identifying the most appropriate room for labour and delivery, all necessary sterile equipment's placed properly and personal protective equipment's are appropriately positioned, assign and inform all healthcare personnel who will be involved in the patient's care before the patient's arrival. ${ }^{9}$ Encourage the pregnant women that they should be attending the routine antenatal, postnatal and abortion care whenever necessary. If there are any complications, additional care should be provided to the pregnant women. ${ }^{3}$

\section{Role of Nurses in Taking Care of Children}

Encourage and teach the parents to follow the scheduling of visits to the hospital, those who are well they have to attend the clinical services in the morning hours and sick visits in the afternoon. Collaborating with community health care providers to identify separate areas for holding well visits for children ${ }^{9}$. Teach the parents to find out the encouraging ways to ventilate their feelings such as fear, nervousness and depression. Every child is unique she/he is having his or her own way of expressing the emotions to facilitate this process engage them in a creative activity and reading story books. Teach the parents to provide safe and supportive environment for the children to express their feelings. If it is necessary to separate the child from their parents, family members or primary caregivers, make sure that appropriate alternative care is provided with the help of other staffs, social worker or similar, they should regularly follow up on the child ${ }^{7}$.

\section{Role of Nurses in Taking Care of the Elderly}

Older adults, those who are in isolation and having the cognitive impairment they may have nervous, fearful, stressed, agitated, depressed, angry and withdrawn during the outbreak or while in quarantine. To provide psychological support through informal networks like social workers and health care professionals. Educate them in a simple ways the facts about COVID-19 and teach them about how to reduce the risk of infection in their own language to elderly people with/without cognitive impairment as they can understand. The information should be given in the form of writing, pictures and images that may be helpful for elderly in understanding. Encourage their family members and other support networks in providing information and helping older people to practice proper preventive measures to avoid transmission of infection. ${ }^{7}$

\section{Role of Nurses in Research}

Nurses should participate in research; evidence based practice and randomized controlled trials related to COVID-19 disease ${ }^{3}$ As at the time of care of patient's they are undergoing with many real life experiences and other important information, however, that experiences and information can be included to discover the evidence based practice in nursing research.

As COVID-19 is a newly identified disease, effective vaccines and treatments are still in development. Thus, in tackling this newly identified infectious disease, nurses face a 
potential risk of infection as well as potential work-related anxiety and mental health problems. It is important to apply the latest knowledge to protect healthcare professionals and nursing staff who are caring for patients with COVID-19. Healthcare providers must be educated about the dangers of infectious disease, including the proper use of personal protective equipment, proper personal hygiene practices, and related environmental measures.

The lived experiences, strategies, and policies related to confronting, exploring, and managing COVID-19 are important for healthcare professionals and nurses in preventing and managing future outbreaks of infectious diseases like COVID19. In addition to basic and clinical studies, conducting studies on disease-spread prevention and the experience of supporting patients physically and psychologically will be crucial. Examining the effects of administrative strategies for disease-spread prevention in both healthcare and community settings will be valuable. Exploring the impact of cultural differences in the perception and prevention of COVID-19 will be one of the most significant issues related to the spread and management of COVID-19 across countries and cultures. ${ }^{10}$

\section{Roles of Nurses in Continuing Education}

They have to take continuing education and training for self it includes, systematically the use of personal protective equipment, scientific reasons of hand washing, and sterilization of patients' articles and management of exposure. Nurses have to learn self-monitoring for signs and symptoms of COVID-19, self-isolation and report illness head nurse, if it occurs $^{8}$. Nurse need special training to work under such challenging conditions. They have to cover full body with personal protective equipment such as gloves, gown and goggles and it is very difficult to do basic nursing care. It is very essential by nurses to undergo pre-training in how to wear, remove and handle the patients with personal protective equipment, to provide skilled nursing care to the patients. During the COVID -19 pandemic, nurses can come across the critically ill patient, sometimes they may need of cardiopulmonary resuscitation. Timely review of cardiopulmonary resuscitation is required by the nurses.

\section{Role of Nurses in Patient Education}

Nurses have to play an additional responsibility of educating the patients and the general public on how to stay healthy and how to prevent viruses from spreading 5 . Provide adequate information about COVID-19 disease, instruction related to disease prevention including, social distancing, staying in the home, frequent hand washing and clean the frequently touched surfaces areas ${ }^{4}$. Inform the patients there are many ways to stay connected and maintain the social networks with family and friends for example, mobile phone, email, social media and video conference. To manage the stress, try to pay attention to your own desires and feelings, exercise regularly, take enough rest and sleep, eat on time and healthy food. News related to the outbreak of COVID-19 can cause anyone to feel panic. Try to get information at specific times during the day from health professionals, authorized news channels and website related to healthcare system. ${ }^{7}$

\section{Role of Nurses in Prevention and Control of Infection}

When speaking or telling about infection control and prevention in complex healthcare settings is challenging. To educate and facilitate nurses and health care professionals or nurses put into practice safely. Their knowledge and skills should be updated and evidence based. Nurses should act as a role model for their sub-ordinates and teach the patients about the importance of infection control and prevention.

They must have accurate knowledge, sufficient training and education about the infection control and prevention. ${ }^{11}$ Nurses those who are working in the intensive care unit, advanced protections are required during daily nursing care and respiratory precautions are required during respiratory related procedures. To reinforce the prevention of infection and control, the infection control officer or inspectors should be appointed to monitor and supervise the cleanliness, hygiene practice and management of infection prevention and control in the hospital or wards by the health professionals. In this way, some highrisk intensive interventions were identified and improvement measures were implemented promptly. COVID-19-specific precautions were drawn among the team consequently, such as waste management. According to recent reports, not only respiratory specimens, but also serum, urine, and stool specimens might be positive for COVID-19. Even though no further IPC advice was provided, advanced procedures for waste managing were necessary, such as collecting respiratory and non-respiratory wastes in covered containers filled with chlorinated disinfectants and discarding in fastened doublelayered medical waste garbage bags. ${ }^{12}$

\section{Role of Nurses in Documentation and Reporting}

Proper documentation and reporting is very important in addition to all of the routine documentation (vital sign, intake and output, daily medication, etc.) associated with patients, nurses should keep a record of entered and exit from the isolation rooms of a patient who was a possible or known case of COVID-19.13 Nurses follow the following guidelines for the documentation and reporting: Fact - They have to collect adequate information about the patient and whatever care is provided by the nurses it should be accurate. A record should contain subjective, objective and descriptive information collected by the nurses. Accuracy - it should be accurate, so that it will be helpful for other health team members. Completeness - while taking the history or information from the patient each and everything should be recorded, it should be complete and concise. Currentness - present history or care must be documented or reported by the nurses, such as vital sign, administration of medication, preparation for diagnostic procedures, clients present condition and admission, discharge and transfer of the patient. Organization documentation and reporting should be well organized format. Confidentiality - confidentiality should be maintained by the all health team members of the each patient. Nurses have to follow the steps of the nursing process for documentation and reporting: Assessment- assess the condition of the patient, in every shift change at the time of handover, nurses should check the all necessary information related to the patients and electronic medical record is matched timely with the patient record or not.

Planning- information collected from the assessment phase that will help for the plan of care of the patient, so what 
planning done for that particular patient that should be recorded.

Implementation- In this phase the nursing care/ medication or procedures, done for individual patient as prescribed or necessary for the patient. After the intervention, observation, management and clinical procedure should be documented in nurses' notes or progress sheet.

Evaluation- In this phase nurses should monitor the effectiveness of management or care in terms of expected outcomes. ${ }^{14}$

\section{Role of Nurses in the Management of Burnout Syndrome}

Due to shift duty there may be more chances of occurrence of higher stress levels during a pandemic, which can result in burnout syndrome for many nurses. Therefore, nurses must take all the necessary precaution to protect themselves both physically and psychologically. Here some techniques to manage the burnout syndrome during the COVID-19 pandemic. The break is very important when your mind and body is telling you to take breaks. At the time of a pandemic, it is not possible for you to go for vacation, but you can go home and take enough rest and come back to work. Use deep breathing techniques to relax yourself when you feel exhausted with the patient's care. Eat healthy and drink adequate amount of water, which is essential to provide energy and preventing from burnout ${ }^{4}$. Some techniques can be followed by nurses to manage the burnout syndrome while working, write your problems in a paper and try to solve that problem or discuss with seniors. Say no to new assignments or task because already you are exhausted with present workload. If possible delegate your work to your colleague or friends. Set the boundaries of your working time, try to finish your work on time and leave the work place on given time. Seek professional support, whenever feeling so stressed and unable to manage that stress take the guidance from your healthcare organization. Appreciate yourself for your hard work and care for the patients.

\section{Role of Nurses in Breaking of Bad News}

In the health care system, bad news is, those news which is giving the negative perception or psychological problems to patients in the future for his or her related disease condition. "Bad news is information that has an adverse and serious effect on an individual's view of his or her future, noting that bad news is always a subjective appraisal by the individual receiving the news".

Nurses have to play an important role in breaking bad news by providing the proper information to the patient; teach the patient to understand, and cope with the bad news, they have been received or given..$^{15}$ Breaking bad news is one of the difficult tasks for nurses; it requires adequate knowledge about the disease condition, good communication skill and patience and must approach with empathy with all patients involved. Provide comfortable position and optimal environment for the patient and relative before discussing about the any news. After understanding the patient's problem the nurses should prepare answers for all the questions arises from the patient. Whenever trying to break bad news related to disease condition, allow the patient to tell about that disease, what he/she knows about that disease or illness.
However than provide the adequate information with evidences related the disease condition or diagnosis.

\section{Role of Nurses in Critical Care}

It is necessary to understand the clinical manifestations of critical illness in COVID-19. In COVID-19 disease, it appears as asymptomatic to critical illness as the current evidences. Those nurses who are working in the intensive care unit they should be prompt to assist in intubation and understand the principles of airway management. When they are assisting cardiac arrest patients, they have to use full personal protective equipment. While transporting the critically ill patient, it is important to ensure the all necessities equipment used while transportation such as oxygen supply and other emergency medications. Effective communication and coordination is essential in a pandemic response. Information should be disseminated to all health care team in a timely manner, and two-way communication should be established. In addition, ICUs will need to work closely with nursing services, infection control, supplies/engineering/cleaning services and other departments to harmonize efforts for a coordinated response. Family engagement is important as visitors are largely excluded from isolation wards. Effective communication is necessary to address concerns and manage expectations, or to help determine the goals of care in the critically ill. Public relations departments and social workers should be involved to facilitate communication with family members or the public media, to minimize misunderstanding and build trust and confidence in the health care system. ${ }^{16}$

\section{Role of the Nurses as Advocate and Mentor for Patients and Family}

During COVID-19 pandemic some patients are critically ill, for management they are intubated, on a ventilator, sedated and isolated from their caregivers or family members. Communication is difficult, so nurses have to advocate between the family members and patients. They have to perform the vital sign, pain assessment as patients are critically ill and high risk for infection. The patient is immobile due to that there is chances of pressure ulcer or bedsore, so every 2 hours turning the patient is necessary. Consistency from every nurse on every shift is also vital to keep the highest level of standards during an epidemic. Nurses must work as a team and communicate effectively in giving information to the patient and family members. In nursing profession nurses are mentored by the other senior nurses and develop their professional practice, skill and knowledge. They have to apply this knowledge and skill to communicate, mentor the patient and family members. ${ }^{13}$

\section{CONCLUSIONS}

Nurses during COVID-19 pandemic will continue to perform their role by keeping a positive attitude towards the present situation, and by involving themselves in all activities related to patient care. They should constantly learn and implement infection control, new policies, procedures, and manage supplies and isolation units. Nurses are playing a critical role 
in the management of COVID-19 patients and achieving the goals of the health care delivery system. They are not only provide nursing care, but also are coordinating and communicating with the family members and other health care workers to meet the goals of the COVID-19 patients.

Financial or Other Competing Interests: None.

\section{REFERENCES}

[1] Duquesne University: school of nursing. Blog: Nurses Responding to Global Pandemics 2020.

[2] World Health Organization. Coronavirus disease (Covid19) outbreak: rights, roles and responsibilities of health workers, including key considerations for occupational safety and health. Interim guidance March 18, 2020.

[3] World Health Organization. Clinical management of severe acute respiratory infection (SARI) when COVID-19 disease is suspected: second edition (version 1.2). Interim guidance March 13, 2020.

[4] Charles A. The role of nurses in the COVID-19 pandemic. UB University at Buffalo April 10, 2020.

[5] Jordan A. On the front lines: the role of nurses during a pandemic. Provo College April 3, 2020.

[6] Dean E. COVID-19: NICE guidance on community and end of life care nursing older people. Clinical Update Posted April 24, 2020.
[7] World Health Organization: Mental health and psychosocial considerations during the COVID-19 outbreak. March 18, 2020.

[8] Huang L, Lin G, Tang L, et al. Special attention to nurses' protection during the COVID-19 epidemic. Crit Care 2020;24(1):120.

[9] Centers for Disease Control and Prevention. Considerations for inpatient obstetric healthcare settings. April 4, 2020.

[10] Chen SC, Lai YH, Tsay SL. Nursing perspectives on the impacts of COVID-19. J Nurs Res 2020;28(3):e85.

[11] Burnett E. Effective infection prevention and control: the nurse's role, nursing standard, evidence and practice. July 4 , 2018.

[12] Ye L, Yang S, Liu C. Infection prevention and control in nursing severe coronavirus disease (COVID-19) patients during the pandemic. Critical Care 2020;24(1):338.

[13] Stirling B, Hatcher J, Harmston J. Communicating the changing role of a nurse in an epidemic: the example of the MERS-CoV outbreak in Saudi Arabia. Journal of Healthcare Communications 2017;2(3):30.

[14] White L, Duncan G, Baumle W. Foundation of nursing. $3^{\text {rd }}$ edn. Australia: Cengage 2011:173-90.

[15] Warnock C. Breaking bad news: issues relating to nursing practice. This learning module has been accredited by the Royal College of Nursing 2016.

[16] Goh KJ, Wong J, Tien JCC, et al. Preparing your intensive care unit for the COVID-19 pandemic: practical considerations and strategies. Crit Care 2020;24(1):215. 